\title{
Visual acuity and retinal line disruption in ocular trauma
}

\author{
Balakrishnan Madhavan ${ }^{1}$, Sowmiya K.R ${ }^{2, *}$, Rekha ${ }^{3}$, Rajarathinam ${ }^{4}$ \\ ${ }^{\mathbf{1}}$ Associate Professor, Dept. of Ophthalmology, Sree Balaji Medical College and Hospital, Chennai, Tamil Nadu, ${ }^{2}$ Associate \\ Professor, Dept. of Community Medicine, Tagore Medical College, Chennai, Tamil Nadu, ${ }^{3}$ Senior Resident, ${ }^{4}$ Professor and \\ HOD, Dept. of Ophthalmology, Sree Balaji Medical College and Hospital, Chennai, Tamil Nadu, India
}

*Corresponding Author:

Email: krs3012@gmail.com

\begin{abstract}
Purpose: Study to find out relationship between visual acuity and retinal visual line disruption in ocular coherence tomogram (OCT) due to (1) changes in the inner segment and outer segment retinal junction (IS/OS), (2) external limiting membrane (ELM) in the foveal region in ocular blunt injuries and to find out it's prognostic value.

Materials and Methods: Prospective, observational study conducted on 100 eyes of 100 patients who visited the department of ophthalmology with blunt ocular injuries. Patients with associated pre-existing ocular and systemic diseases were excluded. Refraction was done to know the best corrected visual acuity in logarithm of minimum angle of resolution (log MAR) after regression of anterior segment signs. OCT was done to scan and study macular pathology and visual lines. Integrity of IS/OS junction and ELM were studied and analyzed the relationship with visual acuity to consider for prognostic value.

Result: Statistically significant relation was noted between the visual acuity and disruption of lines at retinal IS/OS junction and ELM $(\mathrm{P}<0.001)$. Visual acuity was decreased when there was extensive disruption of retinal lines. There was strong correlation between disruption of IS/OS junction and ELM.
\end{abstract}

Conclusion: The retinal visual line disruption can be used as prognostic indication for future vision.]

Keywords: External limiting membrane, Inner segment outer segment junction, Trauma, Visual acuity.

\section{Introduction}

Accidental trauma to eye is a major cause of blindness in the world. Road traffic accidents are the cause for trauma in all ages especially in adult and in old age. Children are getting injured by playing toys with sharp edges and also by non-supervised use of crackers. Sports injuries occur while playing without proper safety shields. Injuries can be thermal, mechanical or chemical. Cracker and bomb blast injuries can have all the three components. Traumatic injuries can be mild like corneal abrasion or severe one affecting all layers of the eye including retina. Optical coherence tomography (OCT) is a quick, non invasive and reproducible imaging tool for macular lesions and has become an essential part of retina practice. OCT is an optical analogy of ultra sound imaging that uses low coherence interferometry to produce cross-sectional images of retina. It captures optical scattering from the tissue to decode spatial details of tissue microstructures. It uses infrared light from a super-luminescent diode that is divided into two parts: one of which is reflected from a reference mirror and the other is scattered from the biological tissue. The two reflected beams of light are made to produce interference patterns to obtain the echo time delay and their amplitude information that makes up an A Scan. A Scan that are captured at adjacent retinal locations by transverse scanning mechanism are combined to produce a two dimensional image. ${ }^{1}$ Now various types of OCT are evolved from the original time-domain OCT to spectral domain (SDOCT) and swept-source OCT (SSOCT). Retinal thickness is a reproducible and common quantitative measurement that is used to monitor the disease process or treatment response using OCT. ${ }^{2}$ Central subfield thickness has high diagnostic value and it correlates with visual acuity. ${ }^{3}$ All OCT instruments make the internal limiting membrane as the inner retinal border. The outer retinal border could be any one of the hyperreflective outer retinal layers depending on machines. ${ }^{4}$ There for the normative value for retinal thickness is unique to the machine used and the ethnic back ground of the subject. ${ }^{5}$

Ocular trauma is one of the commonest causes of preventable vision loss worldwide. ${ }^{6,7}$ In India, the percentage of population affected by ocular trauma at some point of their lives has been reported to be $2.4 \%$ $3.97 \%$ in various studies. ${ }^{8,9}$ In most of ocular injuries, anterior segment is affected maximum and will be healed in many cases. The subtle posterior segment injuries are difficult to identify and cause irreversible damage. Prognosis of posterior segment injuries is difficult to predict. In recent studies the micro structural defects in the outer retina has been shown to have significant visual prognostic implication in numerous diseases such as diabetic macular edema,${ }^{10}$ age related macular degeneration, ${ }^{11}$ uveitic macular edema, ${ }^{12}$ hereditary retinal dystrophies, ${ }^{13}$ epiretinal membrane ${ }^{14}$ and macular hole. ${ }^{15}$ Very few studies were conducted to find out the prognostic use.

\section{Purpose}

This is a prospective, observational study to find out the relationship between visual acuity and so called retinal "visual line" disruption in ocular coherence tomogram (OCT) due to changes in the inner segment and outer segment junction (IS/OS), ${ }^{2}$ external limiting 
membrane (ELM) in the foveal region in blunt ocular injuries.

\section{Materials and Methods}

Prospective observational study was conducted in 100 eyes of 100 patients over a period of 1 year from Nov 2016 to Nov 2017. The study was done in the department of ophthalmology of a medical college at Chennai, India in collaboration with department of community medicine of another medical college. It is a tertiary eye care centre. Few patients were referred by general practitioners and nearby ophthalmologists after conducting multispeciality medical camps. Patients visited the hospital with complaints of dimness of vision, pain, redness, swelling, watering, glare of vision, photophobia, floaters, lid lag and diplopia due to various types of eye injuries. Age, sex, history, investigations and treatment outcome were noted. Tenets of declaration of Helsinki were followed in this study. Institutional Ethics committee had approved the project. Patients with corneal opacity, previous ocular surgeries, previous uveal and retinal diseases, associated systemic diseases, post traumatic brain injuries were excluded from this study. Uncooperative and non willing patients for follow up were also excluded from this study.

Proper written and informed consent was obtained for the management from the patients or from parents. Refraction under cycloplegia was done to find out best corrected visual acuity (BCVA) and it was converted to logarithm of minimum angle of resolution (log MAR) for statistical analysis. Slit lamp examination, $90 \mathrm{D}$ fundus test, direct and indirect ophthalmoscopy, gonioscopy, Goldmann applanation tonometry were done in co-operative and suitable patients at each visit. Patients were reviewed after 1 day, 5days, 1 week, 1 month, 6months and every year in all co-operative patients. 1 month follow up was done strictly in all patients. Cirrus HD OCT machine (CarlZeiss, Meditec, Dublin CA) was used to scan the macula. Three macular cube scans $(512 \times 128)$ were recorded for all eyes. Repeat OCT was taken after 1 month for all trauma patients.

Patients were divided into 3 groups depending on the integrity of IS/OS junction and ELM based on 3 parameters given below.

Group (1)-Line integrity well maintained: Normal with no line disruption

Group (2)-Line integrity less maintained: Line disruption $<100$ micron

Group (3)-Line integrity disrupted: Line disruption $>100$ micron

Variation of visual acuity with IS/OS junction integrity and variation of visual acuity with ELM integrity were studied. These results were analyzed with visual acuity to find out it's prognostic value.

Statistics: Descriptive statistics were used and the data being presented as percentages, mean and standard deviation. Chi-Square and Fishers exact test were used to test the difference in the proportions. $\mathrm{P}<0.05$ was noted as statistically significant difference.

\section{Results}

Results of prospective non-randomized observational study of 100 eyes of 100 patients with ocular injury were analyzed. Male: Female ratio was $72: 28$. Males were more affected by traumatic ocular injuries. There were 22 patients affected in less than 20 years age group, 50 patients affected in 20-40 age group, 20 patients affected in $40-60$ age group and 8 patients were affected in more than 60 years age group. It showed that the traumatic eye injuries were more in active life period of 20-40 years. The above demographic findings were given in table- $1.75 \%$ of patients were recruited from outpatient department and $25 \%$ of patients were referred by general practitioners and outside ophthalmologists after conducting multidisciplinary medical camps

\section{Table 1: Age and sex distribution of subjects}

\begin{tabular}{|l|c|c|c|}
\hline $\begin{array}{l}\text { Age Group (in } \\
\text { years) }\end{array}$ & Female & Males & Total \\
\hline$<20$ & 10 & 12 & 22 \\
\hline $20-40$ & 13 & 37 & 50 \\
\hline $40-50$ & 4 & 16 & 20 \\
\hline$>60$ & 1 & 7 & 8 \\
\hline Total & 28 & 72 & 100 \\
\hline
\end{tabular}

There were statistically significant differences between visual acuity and IS/OS integrity well maintained, less maintained and disrupted group (Mean visual acuity \pm SD in log MAR in 3 groups being $0.50 \pm$ $0.12,1.03 \pm 0.06$ and $1.25 \pm 0.11$ with $\mathrm{P}(<0.001)$. There was statistically significant difference between visual acuity and ELM integrity well maintained, less maintained and disrupted groups (Mean visual acuity \pm $\mathrm{SD}$ in $\log$ MAR in the 3 groups being $0.32 \pm 0.06,0.86$ \pm 0.1 and $1.19 \pm 0.12$ respectively with $\mathrm{P}<0.001)$. Very strong correlation was observed $(\mathrm{R}=0.882)$ between ELM and IS/OS integrity of same patient. After 1 month of treatment, the decreasing visual acuity was noted with more amount of disruption of retinal line. This is depicted in table- 2 and table 3 .

Table 2: Variation of visual acuity with integrity of is/os junction

\begin{tabular}{|l|c|c|c|}
\hline & $\begin{array}{c}\text { IS/OS } \\
\text { Well } \\
\text { Maintained }\end{array}$ & $\begin{array}{c}\text { IS/OS } \\
\text { Less } \\
\text { Maintained }\end{array}$ & $\begin{array}{c}\text { IS/OS } \\
\text { Distributed }\end{array}$ \\
\hline (1) Visual & $0.50 \pm$ & $1.03 \pm$ & $1.24 \pm$ \\
Acuity (in & 0.12 & 0.06 & 0.11 \\
log MAR) & & $<0.001$ & $<0.001$ \\
\hline (2) P-value & & \multicolumn{2}{|l}{} \\
\hline
\end{tabular}


Table 3: Variation of visual acuity with integrity of elm

\begin{tabular}{|l|c|c|c|}
\hline & $\begin{array}{c}\text { ELM Well } \\
\text { Maintained }\end{array}$ & $\begin{array}{c}\text { ELM Less } \\
\text { Maintained }\end{array}$ & $\begin{array}{c}\text { ELM } \\
\text { Disrupted }\end{array}$ \\
\hline (1)Visual & $0.32 \pm$ & $0.86 \pm$ & $1.19 \pm$ \\
$\begin{array}{l}\text { Acuity (in } \\
\text { log MAR) }\end{array}$ & 0.06 & 0.1 & 0.12 \\
\hline (2)P-value & & $<0.001$ & $<0.001$ \\
\hline
\end{tabular}

\section{Discussion}

Retinal anatomy and functions are now well studied with new equipment's due to advanced technology. OCT is now an integral tool of ophthalmology to delineate retinal pathologies. HD OCT machines are newer machine and is helpful to get explicit resolution of various layers of retina. The four hyper-reflective horizontal lines seen on macular OCT with breach in lines shows an abnormal macular function. These retinal lines include from bottom upwards, the retinal pigment epithelium (RPE), the cone outer segment tip (COST) line, the inner segmentouter segment (IS/OS) junction and the external limiting membrane (ELM). In various studies, these lines have been shown to have visual and prognostic significance in numerous diseases. ${ }^{10-15}$ These lines are believed to correspond to the ultra structure of the photoreceptor cells, hence a break in the foveal area is usually associated with permanent damage to the visual functions of this area. However the exact microscopic localization of these lines has been a matter of much debate, the recently it has been shown in studies that the IS/OS junction actually represents the ellipsoid zone of the inner segment. ${ }^{16}$ Whatever the exact anatomical location of these lines, their break has been consistently reported to be associated with poor vision in many diseases. Post vitreo retinal surgery integrity of retina has also been shown to have significant correlation with visual acuity post operatively. ${ }^{17}$

After blunt trauma, some studies have been done to find out the role of these lines among those having commotio retinae. ${ }^{18-24}$ Generally these patients, reveal an increase in reflectivity/intensity of the IS/OS junction, however some studies have also reported loss or attenuation of these lines. ${ }^{18,22,23}$ Appearance of these lines have sometimes been reported to return to normal ${ }^{19,22,24}$ although variable visual recovery is seen to be correlated to the region of the initial disruption in these patients. ${ }^{18,23}$ These latter results are consistent with the original findings of Sipperley et al; who hypothesized that the resultant visual loss in commotio retinae may be determined by the amount of photoreceptor damage occurring during the initial trauma. ${ }^{25}$

The study was conducted on patients with ocular trauma who had no associated general diseases and previous eye diseases. OCT was done twice, once at the time of initial consultation and another after 1 month. We were unable to measure the visual acuity at the beginning due to many unstable condition in anterior and posterior segments of eye including hyphaema, corneal abrasion, uveitis, cataract and vitreous hemorrhage. But final visual acuity was reached after stabilization of all above conditions after 1month, and vision was very well correlated with integrity of IS/OS junction and ELM integrity as revealed by disruption of visual lines in OCT. Therefore these visual lines could be used as prognostic factors for the future visual acuity. Length and width of disruption of lines and subfoveal and juxta foveal location presentations also have prognostic value.

There are merits and demerits for our study. The strength is the reasonable high number of patients (100) and the use of high tech ultra OCT. Limitations are the non-randomized study nature and the smaller follow up duration (1month). Larger trial with larger sample size for higher duration may yield better results. Studies with the help of new better evolving machines may help us to elucidate the secrets further.

\section{Conclusion}

The role of retinal lines was studied on patients after ocular blunt trauma. The prognostic role of IS/OS junction and ELM were studied on a larger sample as much as 100 patients. Our study revealed the decreasing visual acuity on increasing disruption of retinal lines. Visual acuity at the time of presentation has no much value on prognosis of vision. But the retinal status after settlement of ocular pathologies, the status of retinal lines, really indicate the prognosis of vision.

\section{Financial Support and Sponsorship: Nil}

\section{Conflicts of Interest: Nil}

\section{References}

1. Huang D, Swanson EA, Lin CP, Schuman JS, Stinson WG, Chang W, et al. Optical coherence tomography. Science 1991;254:1178-81.

2. Diabetic Retinopathy Clinical Research Network Writing Committee, Bressler SB, Edwards AR, Chalam KV, Bressler NM, Glassman AR, et al. Reproducibility of spectral-domain optical coherence tomography retinal thickness measurements and conversion to equivalent time-domain metrics in diabetic macular edema. JAMA Ophthalmol 2014;132:1113-22.

3. Diabetic Retinopathy Clinical Research Network, Browing DJ, Glassman AR, Aiello LP, Beck RW, Brown $\mathrm{DM}$, et al. Relationship between optical coherence tomography-measured central retinal thickness and visual acuity in diabetic macular edema. Ophthalmology 2007; 114:525-36.

4. Wolf-Schnurrbusch UE, Ceklic L, Brinkmann CK, Iliev ME, Frey M, Rothenbuehler SP, et al. Macular thickness measurements in healthy eyes using six different optical coherence tomography instruments. Invest Ophthalmol Vis Sci 2009;50:3432-7.

5. Girkin CA, McGwin G Jr. Sinai MJ, Sekhar GC, Fingeret M, Wollstein G, et al. Variation in optic nerve and 
macular structure with age and race with spectral-domain optical coherence tomography, Ophthalmology 2011;118:2403-8.

6. Katz J, Tielsch JM. Lifetime prevalence of ocular injuries from the Baltimore Eye Survey. Arch Ophthalmol. 1993; 111:1564-8.

7. Schein OD, Hibberd P, Shingleton BJ, Kunzweiler T, Frambach DA, Seddon JM, et al. The spectrum and burden of ocular injury. Ophthalmology 1988;95:300-5.

8. Vats S, Murthy GVS, Chandra M, Gupta SK, Vashist $\mathrm{P}$,Gogoi M. Epidemiological study of ocular trauma in an urban slum population in Delhi, India. Indian journal of Ophthalmology. 2008;56(4):313-316.

9. Dandona L, Dandona R, Srinivas M, John RK, McCarty CA, Rao GN. Ocular trauma in an urban population in southern India: The Andhra Pradesh Eye Disease study. Clin Exp Ophthalmol.2000;28:350-6.

10. Yinchen Shen, Kun Liu, and Xunxu. Correlation between Visual Function and Photoreceptor integrity in Diabetic Macular Edema: Spectral-Domain Optical Coherence Tomotography. Current Eye Research.2016;3:391-399.

11. Yoon Hyung Kwon, Dong Kyu Lee, Hyung Eun Kim, Oh Woong Kwon. Predictive Findings of Visual Outcome in Spectral Domain Optical Coherence Tomography after Ranibizumab Treatment in Age-related Macular Degeneration. Korean J Ophthalmol 2014;28(5):386-392.

12. Paolo Tortorella, EnzoD' Ambrosio, Ludovico Iannetti, Federica De Marco, and Maurizio La Cava. Correlation between Visual Acuity, Inner Segment/Outer Segment Junction, and Cone Outer Segment Tips Line Integrity in Uveitic Macular Edema. Bio Med Research International. Volume 2015, Article ID 853728.

13. Isabelle Meunier, Carl Arndt, Xavier Zanlonghi, Sabine Defoort-Dhellemmes, Isabelle Drumare, Martine MaugetFaysse, Benjamin Wolff, Aude Affortit, Christian Hamel and Bernard Puech (2012). Spectral-Domain Optical Coherence Tomography in Hereditary Retinal Dystrophies, Selected Topics in Optical Coherence Tomography, Dr. Gangjun Liu (Ed.), ISBN:978-953-510034-8, In Tech.

14. Inoue M. Correlation between the morphology of the IS/OS junction and functional outcomes in patients with idiopathic epiretinal membrane. Nihon Ganka Gakkai Zasshi. 2012 Nov;116(11):1029-36.

15. Shao Q, Xia H, Heussen FM, Ouyang Y, Sun X, Fan Y. Postoperative anatomical and functional outcomes of different stages of high myopia macular hole. BMC Ophthalmol. 2015 Aug 7;15:93.

16. Spaide RF, Curcio CA. Anatomical correlates to the bands seen in the outer retina by optical coherence tomography: Literature Review and Model. Retina (Philadelphia, Pa). 2011;31(8):1609-1619.

17. Matsumiya W, Kusuhara S, Shimoyama T, Honda S, Tsukahara Y, Negi A. Predictive value of preoperative optical coherence tomography for visual outcome following macular hole surgery: effects of imaging alignment. Jpn J Ophthalmol. 2013 May;57(3):308-15.

18. Souza-Santos F, Lavinsky D, Moraes NS, et al. Spectraldomain optical coherence tomography in patients with commotio retinae. Retina.2012;32:711-718.

19. Oh J, Jung JH, Moon SW, et al. Commotio retinae with spectral- domain optical coherence tomography. Retina. 2011;31:2044-2049.

20. Sony P, Venkatesh P, Gadaginamath S, Garg SP. Optical coherence tomography findings in commotio retinae. Clin Exp Ophthalmol. 2006;34:621-623.
21. Meyer CH, Rodrigues EB, Mennel S. Acute commotio retinae determined by cross-sectional optical coherence tomography. Eur J Ophthalmol.2003;13:816-818.

22. Park JY, Nam WH, Kim SH, et al. Evaluvation of the central macula in commotio retinae not associated with other types of traumatic retinopathy. Korean $J$ Ophthalmol.2011;25:262-267.

23. Ahn SJ, Woo SJ, Kim KE, et al. Optical coherence tomography morphologic grading of macular commotio retinae and its association with anatomic and visual outcomes. Am J Ophthalmol. 2013;156: 994-1001.

24. Saleh M, Letsch J, Bourcier T, et al. Long-term outcomes of acute traumatic maculopathy. Retina. 2011;31:20372043.

25. Sipperley JO, Quigley HA, Gass DM. Traumatic retinopathy in primates. The explanation of commotio retinae. Arch Ophthalmol. 1978;96:2267-2273. 